\title{
Transformation plasmonics
}

\author{
Muamer Kadic ${ }^{1}$, Sébastien Guenneau ${ }^{1}$, \\ Stefan Enoch ${ }^{1}$, Paloma A. Huidobro ${ }^{2}$, \\ Luis Martín-Moreno ${ }^{3}$, Francisco J. García-Vidal ${ }^{2}$, \\ Jan Renger ${ }^{4}$ and Romain Quidant ${ }^{4,5, *}$ \\ ${ }^{1}$ Institut Fresnel, CNRS, Aix-Marseille Université, Campus \\ Universitaire de Saint-Jérôme, 13013 Marseille, France \\ ${ }^{2}$ Departamento de Física Teorica de la Materia Condensada, \\ Universidad Autónoma de Madrid, E-28049 Madrid, Spain \\ ${ }^{3}$ Instituto de Ciencia de Materiales de Aragon and \\ Departamento de Fisica de la Materia Condensada, ICMA- \\ Universidad de Zaragoza, Zaragoza 50009, Spain \\ ${ }^{4}$ ICFO-Institut de Ciencies Fotoniques, Mediterranean \\ Technology Park, 08860 Castelldefels (Barcelona), Spain \\ ${ }^{5}$ ICREA-Institució Catalana de Recerca i Estudis Avançats \\ 08810 Barcelona, Spain, e-mail: romain.quidant@icfo.es
}

*Corresponding author

\begin{abstract}
Surface plasmons polaritons (SPPs) at metal/dielectric interfaces have raised lots of expectations in the on-going quest towards scaling down optical devices. SPP optics offers a powerful and flexible platform for real two-dimensional integrated optics, capable of supporting both light and electrons. Yet, a full exploitation of the features of SPPs is conditioned by an accurate control of their flow. Most efforts have so far focused on the extrapolation of concepts borrowed from guided optics. This strategy has already led to many important breakthroughs but a fully deterministic control of SPP modes remains a challenge. Recently, the field of optics was stimulated by a novel paradigm, transformation optics, which offers the capability to control light flow in any desired fashion. While it has already significantly contributed to the design of metamaterials with unprecedented optical properties, its versatility offers new opportunities towards a fully deterministic control of SPPs and the design of a new class of plasmonic functionalities. Here, we review recent progress in the application of transformation optics to SPPs. We first briefly describe the theoretical formalism of transformation plasmonics, focusing on its specificities over its three-dimensional optical counterpart. Numerical simulations are then used to illustrate its capability to tame SPP flows at a metal interface patterned with a dielectric load. Finally, we review recent experimental implementations leading to unique SPP functionalities at optical frequencies.
\end{abstract}

Keywords: cloaking; metamaterial; plasmonics; surface plasmon polariton; transformation optics; transformation plasmonics.

\section{Introduction}

Surface plasmon polaritons (SPPs) are surface waves [1-3] propagating along the interface between two media, usually a dielectric and a metal. Their field distribution extends into both media and is evanescent in the direction perpendicular to the interface. In the case of a homogeneous flat metallic film, SPP modes are delocalized over the plane. The control of SPPs flow has so far been mostly based on concepts directly inspired from integrated optics with dielectric waveguides. The most intuitive approach consists of introducing geometrical boundaries by patterning the metal layer. This has for instance enabled subwavelength guiding of SPPs along strips [4, 5], V-grooves and slots [6-8] as well as adiabatic focusing $[9,10]$.

Alternatively, controlling SPP modes can be done by spatially engineering its effective refractive index by either using a finite dielectric load [11-15] or structuring the metal surface. For instance, Pendry et al. showed in 2004 that one can manipulate surface plasmon ad libitum via homogenization of structured surfaces [16]. This powerful concept has enabled the experimental demonstration of broadband extraordinary transmission in the visible regime by means of plasmonic checkerboards [17, 18].

More recently, the field of metamaterials has inspired a new form of SPP control. In 2008, Smolyaninov et al. achieved a noticeable reduction in the scattering of SPPs incident upon a cloak consisting of polymethylmethacrylate (PMMA) at a wavelength of $532 \mathrm{~nm}[19,20]$. More recently, Baumeier et al. demonstrated theoretically that it is possible to reduce significantly the scattering of an object by an SPP at a wavelength of $633 \mathrm{~nm}$ when it is surrounded by two concentric rings of point scatterers [21].

The recent proposal of transformation optics (TO) [2230] has brought a novel way of controlling flows of photons in any desired way. The TO concept has for instance permitted us to achieve unique light control including the concept of invisibility [31-44], lenses [45-48], beamshifter, splitters and bends [49-54], or more exotic applications such as electromagnetic wormholes [55, 56], hidden pathways [57, 58], or optical black holes [59-66]. Moreover TO can further be used to completely change the appearance of an object thereby creating an illusion [67-70], or to enable subwavelength imaging via hyperlenses that transform the evanescent waves [71-75]. TO often takes advantage of the field of metamaterials [76-78] to implement complex electromagnetic parameters. Metamaterials are artificial materials made up of subwavelength constituents that are designed to effectively mimic a prescribed response to electromagnetic fields and that often make use of plasmonic structures such as plasmonic nanomaterials, multilayer SPPs and so on [79]. On the other 
hand, we note in passing that plasmonic routes to cloaking based on core shell resonances induced by a coating of low refractive index (scattering cancellation [80]) and negative refractive index (external cloaking [81]) have also been proposed.

Whereas at first TO was applied to plane waves, it has been recently shown that TO can also be used to effciently mould the flow of surface plasmon polaritons (SPPs) at metaldielectric interfaces [82-88]. Using TO to manipulate SPPs is a very general technique that has already led to a wide variety of proposals for plasmonic elements which might even be based on single-atom-thin graphene layers [89]. Moreover, TO has also been applied to analytically handle localized surface plasmons in metallic nanostructures [90-94] including non-local effects [95].

In the present article we review the state of the art in the exciting field of transformation plasmonics. After presenting how TO can be extended to SPP, we illustrate numerically its capability to fully control the SPP flow by loading the metal surface with a patterned dielectric film. We finally review recent experimental implementations in which transformation plasmonics is applied to broadband plasmonic invisibility and focusing.

\section{Formalism of transformation plasmonics}

\subsection{Transformation optics: coordinate changes in Maxwell's equations}

Conceptually, TO is equivalent to warp space with the aim to control trajectories of light rays. While space can not be transformed in this manner, TO alternatively makes coordinates transformations, which in practice, provide the local optical properties to be fulfilled by the distorted space to achieve the desired light flow. This is a subject which has received steadily more attention from 2006 onwards, thanks to the paradigm of the invisibility cloak. However, this topic got started in the mid 1990s with the original aim to reduce the complexity of computational problems [96-98]. The cornerstone of TO was to map, for instance, an infinite domain on a finite region of space, which would then be considered in the numerical package. The point of view of TO was central in the development of finite elements models for twisted microstructured fibers [99] as their modeling involves twisted perfectly matched layers.

As we start with a given set of equations on a given domain, it seems at first sight that we have to map this domain on a new one. Nevertheless, it is the opposite that has to be done, as shown in Figure 1. On the original domain, see Figure 1B, the equations are described in a particular coordinate system $(x, y)$ (taken here by default to be Cartesian coordinates). We want to establish a one-to-one correspondence with a new domain where we use a general (possibly non-orthogonal) coordinate system $(u, v)$, see Figure 1, panels (A) and (D), (which correspond respectively to a rotation and a compression of some region of the original domain). More precisely, consider a map from the curvilinear coordinate system $(u, v)$ to the initial one

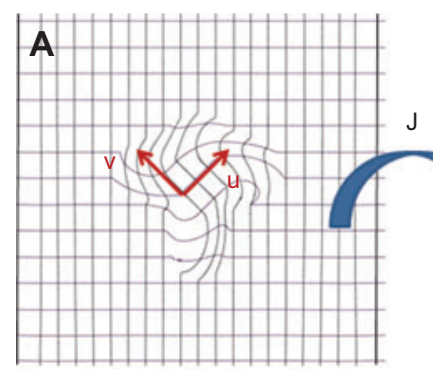

$$
\begin{aligned}
& \left\{\begin{array}{l}
d x=\frac{\partial x}{\partial u} d u+\frac{\partial x}{\partial v} d v \\
d y=\frac{\partial y}{\partial u} d u+\frac{\partial y}{\partial v} d v
\end{array}\right. \\
& \Leftrightarrow\left(\begin{array}{l}
d x \\
d y
\end{array}\right)=J\left(\begin{array}{l}
d u \\
d v
\end{array}\right)
\end{aligned}
$$
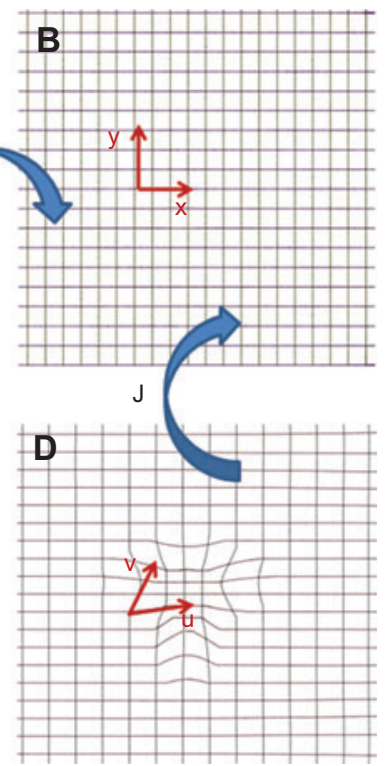

Figure 1 Principle of transformation optics: (A) Transformed domain in coordinate system $(u, v)$ corresponding to a rotation of domain in (B); (B) Original coordinate system $(x, y)$; (C) Jacobian matrix of $x(u, v)$ and $y(u, v)$, which are explicit functions of the coordinates $u$ and $v$. (D) Transformed domain in coordinate system ( $u$, $v$ ) corresponding to a compression of domain in (B); seems at first sight that we have to map the coordinates in (B) on the new ones in (A) [resp. (D)]. Nevertheless, it is the opposite that has to be done: the new coordinate system in (A) [resp. (D)] is mapped on the initial one in (B).

$(x, y)$ given by the transformation $x(u, v)$ and $y(u, v)$. Here, we must point out that, we are mapping the transformed domain and coordinate system [Figure 1 (A) or (D)] onto the initial one [Figure 1 (B)] with Cartesian coordinates. This change of coordinates is characterized by the transformation of the differentials through the Jacobian matrix $\mathbf{J}$ described in Figure 1(C).

Importantly, such a geometric transformation also affects the material properties in the transformed coordinates which are encompassed in the constitutive Maxwell's equations. More precisely, in electromagnetism, changes of coordinates amount to replacing the different materials (often homogeneous and isotropic, which corresponds to the case of scalar piecewise constant permittivity and permeability) by equivalent inhomogeneous anisotropic materials. Mathematically speaking, the permittivity and permeability are rank-2 anisotropic heterogeneous tensors in the transformed coordinates:

$\hat{\varepsilon}^{\prime}=\varepsilon_{0} \mathbf{T}^{-1}$, and $\hat{\mu}=\mu_{0} \mathbf{T}^{-1}$ where $\mathbf{T}=\mathbf{J}^{T} \mathbf{J} / \operatorname{det}(\mathbf{J})$.

$\mathbf{J}$ is the Jacobian matrix of the transformation, see Figure 1, and $\mathrm{T}$ is the metric tensor in the transformed coordinates. In Eq. 1, the right hand sides involve matrix products where the matrix associated with the second rank tensor $\mathbf{T}$ contains the coeffcients of its representation in the initial Cartesian coordinate system $(x, y)$.

Let us make use of the invisibility cloak to illustrate the concept of TO. One way to conceal a region of space 
is mapping a disc or radius $r<R_{2}$ onto a corona $R_{1}<r^{\prime}<R_{2}$, as shown in panels (A) and (B) of Figure 2 (note that $r$ is replaced by $r^{\prime}$ in the corona, since we have now a stretched radial coordinate). This mapping can be done by means of a linear transformation, $r^{\prime}=R_{1}+r\left(R_{2}-R_{1}\right) / R_{2}$, as proposed by Pendry et al. [22] (a transformation formally used in the context of inverse problems in tomography [100]). The effect of this transformation on the metric of space is shown in Figure 2(C): the metric is compressed in the corona surrounding the concealed region in panel (B). The obtained matrix provides the new coeffcients of the tensor corresponding to the equivalent material in Figure 2 (B). Note that the transformed grid in panel (B) does not have right angles as the one in panel (A): the transformed material is anisotropic, as can be clearly seen in Figure 2 (C). It should be noted that the off-diagonal elements of $\mathbf{T}$ are identical and therefore $\mathbf{T}$ can be diagonalized. Importantly, all diagonal elements have positive entries (negative values in the off-diagonal elements is just an effect of the rotation required to express $\mathbf{T}$ in a diagonal basis), however they either vanish or become extremely large on the inner cloak boundary, which is consistent with the fact that detoured waves, as shown in Figure 2(D) for a source located in the close neighborhood of a two-dimensional cloak, have to be accelerated in the cloak in order to catch up with their wavefront outside the cloak.

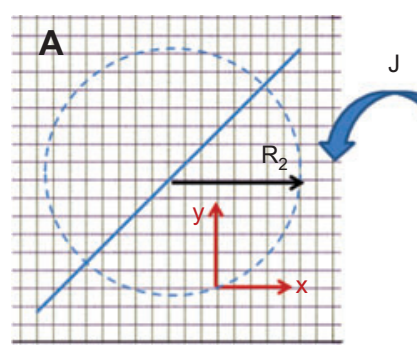

C

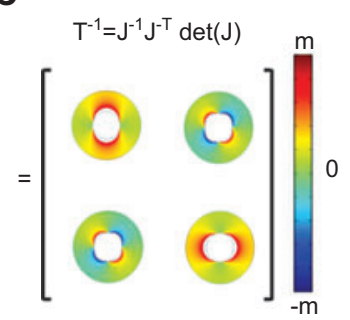

D

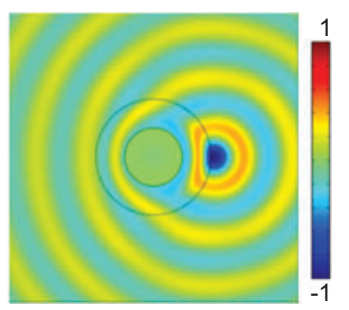

Figure 2 Concept of metric tensor with an example: (A) Metric of virtual space in original coordinate system $(x, y)$; (B) Metric of physical space in transformed coordinate system $(u, v)$, illustrated by Pendry's paradigm of a hole in the metric (invisibility region) surrounded by a corona of compressed metric (invisibility cloak). (C) Spatial distribution of the entries of the metric tensor (prescribing the permittivity and permeability) inside the cylindrical cloak. (D) Plot of the (only non-vanishing) longitudinal component of magnetic field; note that $m$ defines an (arbitrarily large) order of truncation of the color scale, and white regions in (C) correspond to singular values of tensor entries greater than $m$ (outside the color scale).

\subsection{From transformation optics to transformation plasmonics}

Let us now apply TO to the control of surface wave trajectories. A few words on the mathematical setup are necessary in order to understand the specificity of the transformation plasmonics design, which involves anisotropic heterogeneous media. To simplify the discussion, we consider a transverse magnetic ( $p$-polarized) SPP propagating in the positive $x$-direction at a flat interface $z=0$ between a (isotropic homogeneous) metal $(z<0)$ and a transformed medium $(z>0)$ described by diagonal tensors of relative permittivity and permeability $\hat{\varepsilon}^{\prime}=\operatorname{diag}\left(\varepsilon_{x x, 2}\right.$, $\left.\varepsilon_{y y, 2}, \varepsilon_{z z, 2}\right)$ and $\hat{\mu}^{\prime}=\operatorname{diag}\left(\mu_{x x, 2}, \mu_{y y, 2}, \mu_{z z, 2}\right)$ :

$\left\{\begin{array}{l}\mathbf{H}_{2}=\left(0, H_{y, 2}, 0\right) \exp \left\{i\left(k_{x, 2} x-\omega t\right)-k_{z, 2} z\right\}, z>0, \\ \mathbf{H}_{1}=\left(0, H_{y, 1}, 0\right) \exp \left\{i\left(k_{x, 1} x-\omega t\right)+k_{z, 1} z\right\}, z>0 .\end{array}\right.$

Note here that $\operatorname{Re}\left(k_{z, 1}\right)$ and $\operatorname{Re}\left(k_{z, 2}\right)$ are strictly positive in order to maintain evanescent fields above and below the interface $z=0$.

For this field to be solution of Maxwell's equations, continuity of its tangential components is required across the interface $z=0$, which translates into mathematics as $k_{x, 1}=k_{\mathrm{x}, 2}=k_{x}$ and this in turn leads us to a local dispersion relation for a p-polarized SPP:

$k_{z, i}=\sqrt{\varepsilon_{x x, 2}\left(\frac{k_{x}^{2}}{\varepsilon_{z z, 2}}-\mu_{y y, 2}\left(\frac{\omega}{C}\right)^{2}\right)}, j=1,2$,

$\frac{k_{z, 1}}{\varepsilon_{1}}+\frac{k_{z, 2}}{\varepsilon_{x x, 2}}=0$.

Here $c$ is the speed of light in vacuum, $\varepsilon_{1}(x, y)>1(z>0)$, and $\varepsilon_{2}$ is the usual Drude form in the metal for $z<0$. Actually, we note that since $k_{z, 1}$ and $k_{z, 2}$ are strictly positive, SPPs can only exist if $\varepsilon_{1}$ and $\varepsilon_{x x, 2}$ are of opposite signs.

Equation 3 can be recast in a more compact form [82]:

$k_{x}=\frac{\omega}{C} \sqrt{\frac{\varepsilon_{z z, 2} \varepsilon_{1}\left(\mu_{y y, 2} \varepsilon_{1}-\varepsilon_{x x, 2}\right)}{\varepsilon_{1}^{2}-\varepsilon_{x x, 2} \varepsilon_{z z, 2}}}$,

which should be satisfied for SPPs to be able to propagate on a flat interface between a metal substrate and a transformed medium. Indeed, SPPs are bound to the interface, hence, they do not belong to the radiative spectrum.

Invisibility carpets were an early proposal of transformation plasmonics [82-88] based on ground-plane cloaks [25]. As shown in Figure 3, the invisibility carpet flattens the SPPs wavefronts reflected by a curved cylindrical mirror on a flat metal surface. The mapping that makes this possible is given by $x^{\prime}=x\left[x_{2}(y)-x_{1}(y)\right] / x_{2}(y)+x_{1}(y)$, which maps the region $0<x<x_{2}(y)$ onto $x_{1}(y)<x^{\prime}<x_{2}(y)$ for $a<y<b$ and $z>0$. The vertical coordinate $z$ appears trivially in the definition of the carpet as it is of cylindrical geometry, but more complex geometries can be explored, and for these we refer to the next 

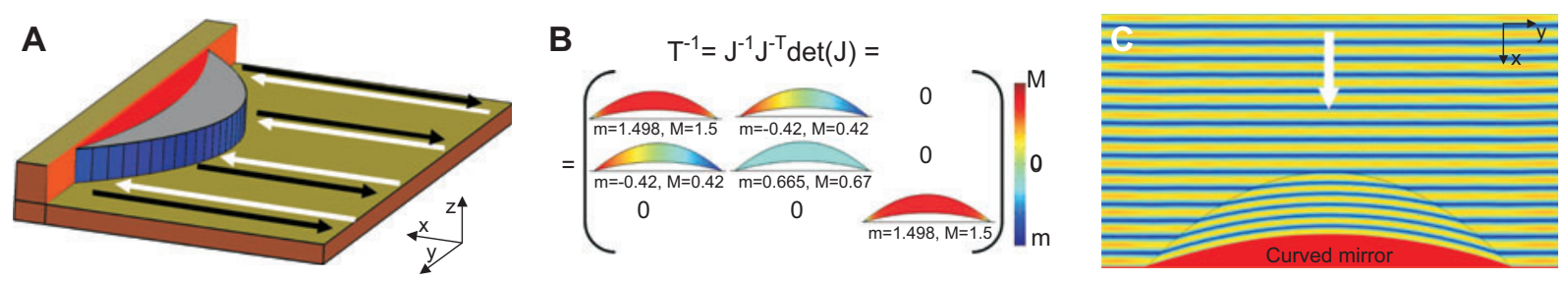

Figure 3 Controlling SPPs on a flat metal surface: (A) SPP incident upon a curved mirror on a metal surface with an invisibility carpet (transformed region in blue) undergoes the same scattering as it would produced by a flat mirror; (B) spatial distribution of entries of the metric tensor (prescribing the permittivity and permeability) inside the cylindrical carpet; (C) 2D plot of the real part of the $y$ component of magnetic field for a SPP incident from above at $\lambda=700 \mathrm{~nm}$ on the transformed medium with permittivity and permeability as in (B).

section. The effect of the mapping on the spatial distribution of the metric tensor is shown in panel (B) of this figure, and the corresponding plot of the $y$-component of the magnetic field for a SPP field incident upon such a plasmonic carpet is shown in panel (C). Interestingly, this metamaterial can be designed with dielectric conical pillars and further tested numerically for different wavelengths such as at $\lambda=700 \mathrm{~nm}$ (see Figure 10 E). An experimental demonstration of such theoretical concepts is shown in section IV B.

Now, if a perturbation on the profile of the flat surface is introduced, for instance a bump as in Figure 6, the former dispersion relation Eq. 4 changes and SPPs move to the radiative spectrum and so scattering cannot be avoided. The dispersion relation Eq. 4 becomes slighlty more complex in the case of curved surfaces (see Ref. [87] for a derivation in the weakly curved configuration), which is the bottom line of transformation plasmonics on curved surfaces, first introduced in Ref. [83] and [84]: enabling SPPs to propagate free of scattering on curved surfaces, hence on longer distances, is a great challenge. This case will be treated in the next section.

\section{Design of plasmonic functionalities based on transformation optics}

In this section we exemplify the theory by means of different plasmonic devices that are designed using the TO tools. Here we concentrate on four devices that control the propagation of SPPs at a metal surface: the cylindrical cloak for SPPs [82, 83], the plasmonic right-angle bend [86], the ground-plane cloak for SPPs [83-86] and the plasmonic Luneburg lens $[48,84]$. In order to numerically demonstrate the application of the TO framework to SPPs, we use a finite element method solver [101].

Since SPPs are surface waves propagating along the interface between two media, its field distribution extends into both media and is evanescent in the direction perpendicular to the interface. Therefore, in order to operate over the whole plasmonic field, the expressions for the electromagnetic (EM) material parameters, $\hat{\varepsilon}$ and $\hat{\mu}$, provided by the TO formalism should, in principle, be implemented both in the dielectric and the metal sides of the interface. This is a fabrication challenge when it comes to practical realizations, as TO generally prescribes highly anisotropic and inhomogeneous $\hat{\varepsilon}$ and $\hat{\mu}$. Moreover, since the plasmon field extends over a few tens of nanometers inside the metal only, $\hat{\varepsilon}$ and $\hat{\mu}$ should be manipulated at such length scales within the metal. However, it has been shown $[83,84]$ that manipulating the material properties inside the metal part is not actually required: a simplified version in which only the EM properties of the dielectric side are modified leads to very accurate functionalities. The problem of the anisotropy can be solved by choosing the appropriate coordinate transformations. When conformal and quasiconformal mappings are considered, the resulting EM parameters are isotropic. Therefore, plasmonic devices can be based on implementations of transformation media based only on isotropic dielectrics, allowing the possibility of lowloss and broad-band performances.

\subsection{D Cylindrical cloak}

As a first illustration of the methodology, let us consider a three-dimensional (3D) cylindrical cloak for SPPs traveling along the interface between a metal and a dielectric. The plasmonic cloak is based on the twodimensional (2D) invisibility cloak that was first proposed within the general TO framework [22, 23] and had an early experimental realization [31]. The purpose of the $2 \mathrm{D}$ cloak was to hide to an external observer any object placed inside it, in a circle of radius $R_{1}$, and the cloak itself [see Figure 4 (A)]. This is done by transforming from a Cartesian space where light propagates in straight lines to a space with a hole of radius $R_{1}$ surrounded by a compressed region of radius $R_{2}$. The radial transformation $r^{\prime}(r)=R_{2} /\left(R_{2}-R_{1}\right) r+R_{1}$ is performed in the cloak region, that corresponds to the shell $R_{1}<r^{\prime}<R_{2}$. The EM parameters resulting from the TO approach in a cylindrical basis are $\hat{\varepsilon}^{\prime}=\left(\varepsilon_{r}, \varepsilon_{\theta}\right.$, $\left.\varepsilon_{z}\right)$ and $\hat{\mu}^{\prime}=\left(\mu_{r}, \mu_{\theta}, \mu_{z}\right)$, with:

$$
\begin{aligned}
& \varepsilon_{r}=\mu_{r}=\frac{r^{\prime}-R_{1}}{r^{\prime}} ; \varepsilon_{\theta}=\mu_{\theta}=\frac{r^{\prime}}{r^{\prime}-R_{1}} ; \\
& \varepsilon_{z}=\mu_{z}=\left(\frac{R_{2}}{R_{2}-R_{1}}\right)^{2} \frac{r^{\prime}-R_{1}}{r^{\prime}}
\end{aligned}
$$

When these parameters are implemented in the cloak, a plane wave traveling through it will follow trajectories that surround the hole and the wave will appear to have traveled through empty space. Note that the required permittivity and permeability are anisotropic, inhomogeneous and take singular values at the inner interface of the cloak. 
A
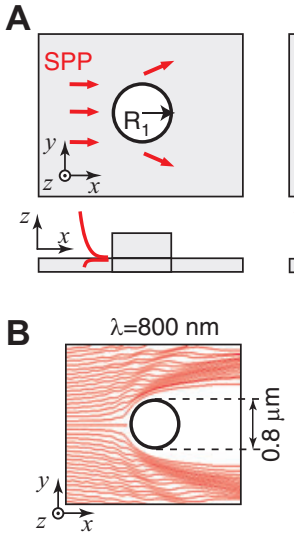
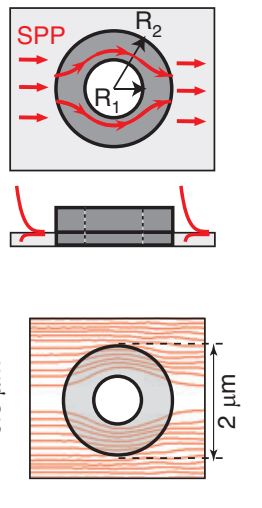

C

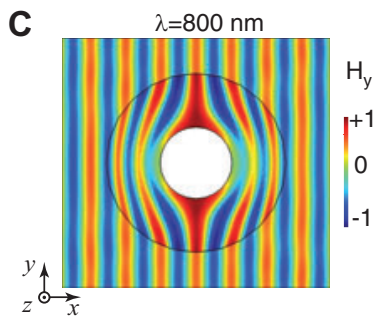

D

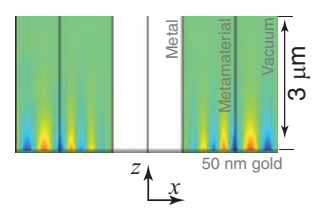

E

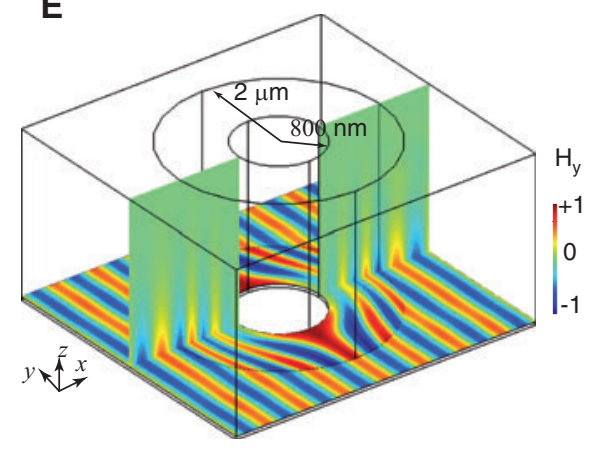

Figure 4 3D cylindrical cloak for SPPs propagating along an air-gold interface. (A) Sketch of the top and side views of the geometry. Left: scattering by a metallic cylinder. Right: the cloak is placed around the cylinder. (B) Power flow from 3D simulations. Left: a SPP experiences high scattering losses when it encounters the bare cylinder. Right: the cloak guides the SPP wave suppressing the scattering losses. (C), (D) and (E) $H_{y}$ field of the SPP when the cylinder is cloaked in a top, side and 3D view, respectively. The height of the cloak is $3 \mu \mathrm{m}$ in the dielectric side and $50 \mathrm{~nm}$ in the metal side.

Let us now discuss the 3D plasmonic cloak, which suppresses the scattering of SPPs propagating along an airgold interface from a metallic cylinder of radius $R_{1}$. As sketched in Figure 4 (A), the SPP is scattered when it encounters the cylinder on top of the gold surface. Designing the 3D cloak involves extending the TO procedure explained above for the 2D cloak to 3D. In particular, the described 2D transformation should be made in an infinite number of planes parallel to the air-gold interface. In order to operate over the whole plasmonic field, we need to cloak the SPP in the dielectric and metal sides, as illustrated in the top right sketch in Figure 4 (A). In the dielectric side, the cloak is a cylindrical shell of radii $R_{1}$ and $R_{2}$ characterized by the EM tensors given by Eq. $5: \hat{\varepsilon}^{\prime}$ and $\hat{\mu}^{\prime}$. The height of the cloak, $h_{d}$, needs to be larger than the decay length of the SPP in vacuum. In the metal side the cloak is a cylindrical shell of the same radii and with a height $h_{m}$ larger than the skin depth. In this case, the EM tensors need to take into account the background material parameters: $\hat{\varepsilon}^{\prime} \cdot \varepsilon_{A u}$, and $\hat{\mu}^{\prime}$. The described cloak, when placed around a metallic cylinder, renders it invisible by suppressing any scattering from it.

Figure 4 shows the results obtained from $3 \mathrm{D}$ simulations of the cylindrical cloak for a SPP at $\lambda=800 \mathrm{~nm}$ traveling on an air-gold interface. The radius of the metallic cylinder is $R_{1}=800 \mathrm{~nm}$ while the outer radius of the cloak is $R_{2}=2000 \mathrm{~nm}$. Panel (B) depicts the power flow streamlines in a top view for two situations: a metallic cylinder scattering the SPP (left sketch) and the cloak guiding the SPP around the metallic cylinder (right sketch). Panels (C) and (D) show a top and a lateral view, respectively, of the $y$ component of the magnetic field of the SPP when the cylinder is cloaked. These images, together with the 3D view of the simulation [panel (E)] show how the SPP field is smoothly guided around the metallic cylinder avoiding any scattering from it.

\subsection{Right angle bend}

As a second example of plasmonic devices designed using TO, we present the right-angle bend for SPPs. This device rotates the propagation direction of an incident SPP by $90^{\circ}$, as shown in the sketch in Figure 5 (A). Similarly to the cylindrical cloak, the design of the 3D rightangle bend is based on 2D transformations parallel to the interface plane. In order to bend the SPP propagation direction from the $x$ direction to the $y$ axis, each transformation consists of mapping a squared region of side $b$ in a Cartesian grid to polar grid with a squared crosssection of the same size. This transformation is given by $r^{\prime}=y, \phi^{\prime}=\frac{\pi}{2 b}(b-x)$ and $z^{\prime}=z$, with $r=\left(x^{2}+y^{2}\right)^{1 / 2}$ and $\phi=\arctan$ $(y / x)$. The following TO parameters are obtained:

$\varepsilon_{r}=\mu_{r} \frac{2 b}{\pi r^{\prime}} ; \varepsilon_{\theta}=\mu_{\theta}=\frac{\pi r^{\prime}}{2 b} ; \varepsilon_{z}=\mu_{z}=\frac{2 b}{\pi r^{\prime}}$

In order to avoid the singularity of these parameters at $r^{\prime}=0$, we limit the radius between $r^{\prime}=a$ and $r^{\prime}=b$. The curvature radius of the bend is defined as $\rho=(a+b) / 2$ and its width by $\Delta=b-a$.

A SPP right-angle bend operating at $\lambda=800 \mathrm{~nm}$ is shown in Figure 5 (A). The SPP propagates in the $x$ direction and enters a bend of curvature radius $\rho=2 \mu \mathrm{m}$. The EM parameters given by Eq. 6, which are anisotropic and inhomogeneous, are implemented in a cylindrical shell comprised between $r^{\prime}=a, r^{\prime}=b, \varphi^{\prime}=0, \phi^{\prime}=90^{\circ}$ in the dielectric side of the interface. From the power flow stream lines (in white) and the shape of the wave-fronts (color scale) it can be interpreted that the SPP field is bended by $90^{\circ}$. The transmittance through the bend, defined as the fraction of the SPP power flow in the exit face compared to the entrance face, is $98 \%$. The lacking $2 \%$ corresponds to the field contained in the metal, which is left untouched.

As we have seen for the plasmonic cloak, TO leads to a perfect functionality when we operate over the whole plasmonic field by introducing a transformation medium in the dielectric and metal sides. However, an experimental realization would require a control of both the dielectric and metal 


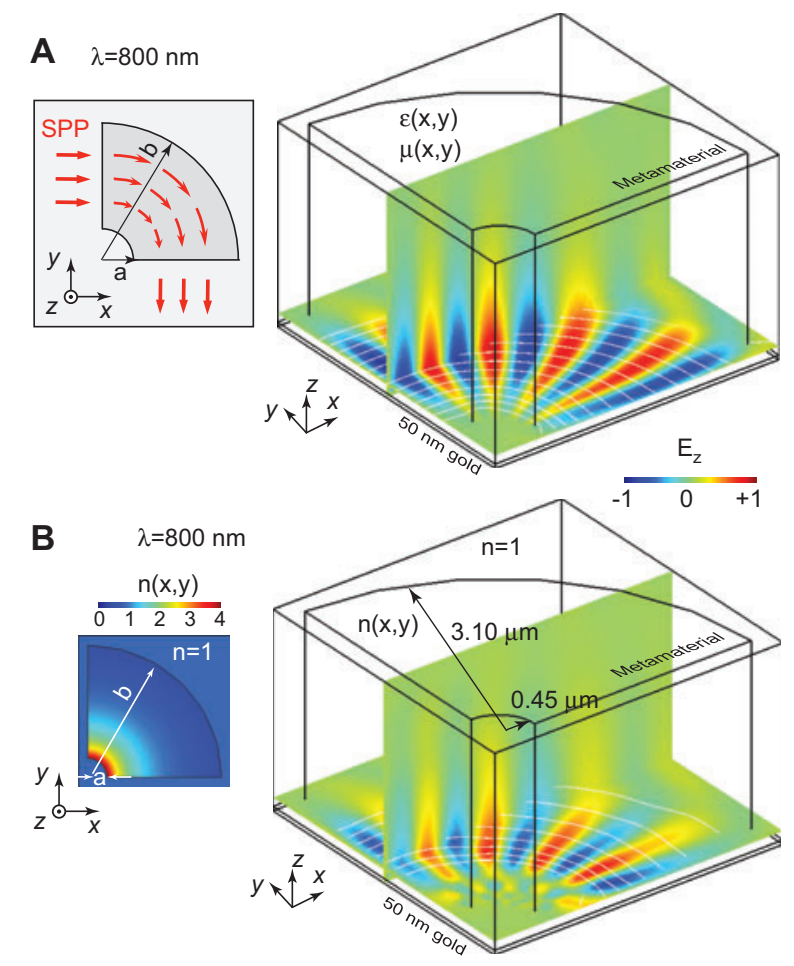

Figure 5 Simulation results for a SPP right-angle bend at $\lambda=800$ $\mathrm{nm}$. The curvature radius of the bend is $\rho=2 \mu \mathrm{m}$ and the SPP has a Gaussian profile of width $\Delta=2.65 \mu \mathrm{m}$. (A) Anisotropic transformation medium. The propagation direction of a SPP is rotated by $90^{\circ}$ by means of a metamaterial with anisotropic $\varepsilon$ and $\mu$. Inset: geometry layout in a top view. (B) Isotropic transformation medium. The SPP bend is characterized by the isotropic transformation medium $n(x, y)$ shown in the inset panel. In both (A) and (B) the color scale plots the $z$ component of the electric field of the SPP and the white lines correspond to power flow stream lines.

EM properties at the nanoscale. This challenge can be circumvented by an approximate approach to TO in which the metal side is left untouched. As we have shown here for the plasmonic bend at $800 \mathrm{~nm}$, manipulating only the dielectric side gives very good results [see Figure 5 (A)]. This model, that is more realistic, has been shown to lead to quasi-perfect performance over broad frequency ranges and for a wide variety of plasmonic devices [86]. The reason for this is that most of the SPP energy is contained in the dielectric since the SPP decay length at this side of the interface is always much larger than in the metal side. Hence, this approximation is more accurate for longer wavelengths, where the skin depth of the metals is almost negligible.

A beam bend with the same functionality as the one we have discussed can also be realized out of isotropic materials. The idea is to use conformal mappings, a specific kind of coordinate transformations that yield grids that are orthogonal, i.e., the angles between coordinate lines are preserved after the mapping. This means that these kind of transformations minimize the anisotropy of the grid. For problems where the two polarizations can be treated separately, such as SPPs, a 2D conformal transformation leads to an isotropic transformation medium. Therefore, a design of the bend based on a conformal transformation replaces the anisotropic EM parameters in Eq. 6 by an isotropic refractive index:

$n(r)=\frac{2 b}{\pi r}$

The refractive index for a bend of the same geometrical parameters as before, which is plotted in the inset panel of Figure 5 (B), varies from 4.46 at the inner radius a to 0.67 at the outer radius $b$. As illustrated in the panel, the plasmonic device is a cylindrical shell comprised between $a<r^{\prime}<b$ and $0<\phi^{\prime}<90^{\circ}$, with a height $h_{d}$, placed on top of the gold surface and characterized by the $n(r)$ profile. The electric field (color scale) and the power flow (white lines) of a SPP traveling through the bend at $\lambda=800 \mathrm{~nm}$ is plotted in Figure 5 (B), showing how the propagation direction of the SPP smoothly rotates by $90^{\circ}$. In this case, the transmittance through the bend is $95 \%$, slightly lower than for the anisotropic bend. The reason for this is that the conformal transformation causes an impedance mismatch at the interfaces of the device (see the inset panel) that results in reflections. This implementation is a more feasible approach for bending the propagation direction of a SPP since it relies on the manipulation of non-magnetic isotropic dielectrics.

\subsection{Ground-plane cloak}

The TO-based approach to the design of plasmonic devices can also be applied to minimizing the scattering to the far-field of SPPs on irregular surfaces. Uneven surfaces result in strong scattering that lead to radiation of the SPP into free-space, as illustrated in Figure 6(A), where a SPP traveling along an air-gold interface encounters an obstacle. Here we model the irregularities of the metal surface as a bump of length $l$ and height $h_{0}$, and with a shape given by $z(x)=h_{0} \cos ^{2}\left(\frac{\pi}{l} x\right)$. We consider a SPP at a wavelength of $700 \mathrm{~nm}$, and a bump of length $l=2 \mu \mathrm{m}$ and height $h_{0}=200 \mathrm{~nm}$. For these parameters, about $15 \%$ the plasmonic energy is scattered by the bump. This scattering can be suppressed by means of a groundplane cloak [25] that, depending on the transformation that we choose can be anisotropic or isotropic. Here we compare both cases.

The design of the anisotropic cloak involves transforming a rectangular region of a Cartesian space sized $l \times h$ into a region of the same shape except that its bottom boundary follows the shape of the bump. The simplest mapping that can be done between these two spaces is a transfinite transformation: $x^{\prime}=x$, $y^{\prime}=y$ and $z^{\prime}=z+h_{0}\left(1-\frac{z}{h}\right) \cos ^{2}\left(\frac{\pi}{l} x\right)$. The transformed coordinate grid when $l=2 \mu \mathrm{m}$ and $h_{0}=200 \mathrm{~nm}$ is depicted with gray lines in Figure 6 (B). When applying the TO procedure, this transformation leads to the following material parameters:

$\hat{\varepsilon}^{\prime}=\hat{\mu}^{\prime}=\frac{1}{\Delta}\left(\begin{array}{ccc}1 & 0 & f_{x z} \\ 0 & 1 & 0 \\ f_{x z} & 0 & f_{z z}\end{array}\right)$ 

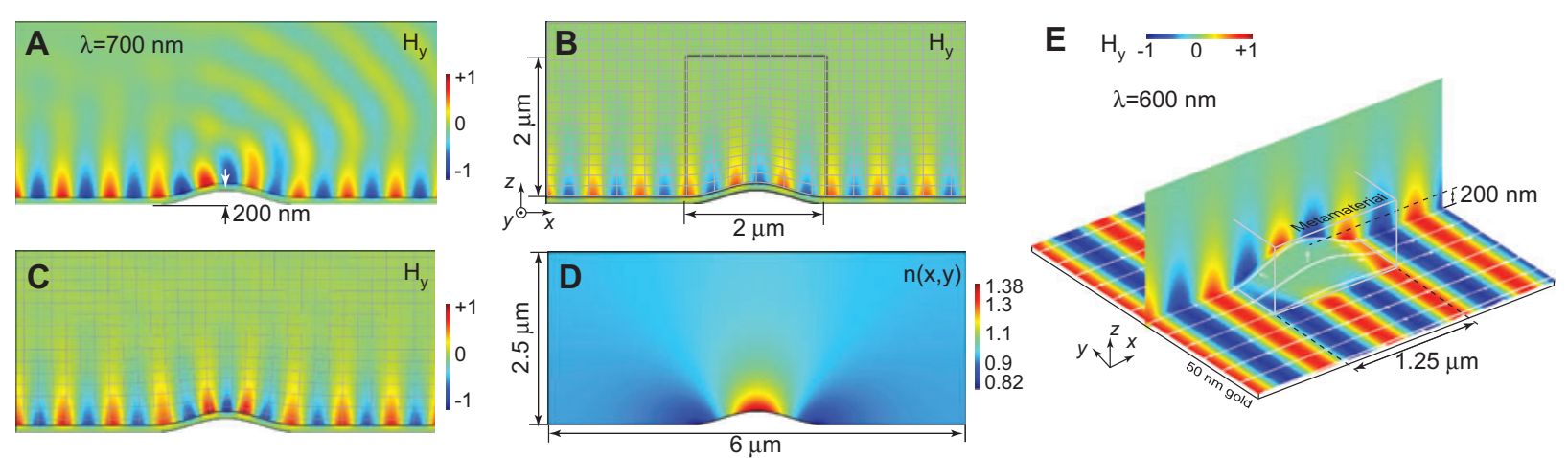

Figure 6 The scattering from a bump on a metal surface can be suppressed by means of a ground-plane cloak. (A) A SPP at $700 \mathrm{~nm}$ is scattered when it encounters a $\cos ^{2}$-shaped bump $200 \mathrm{~nm}$ high and $2 \mu \mathrm{m}$ long in an air-gold interface. The SPP propagates in the $x$ direction and the geometry is invariant in the $y$ direction. (B) Scattering losses are suppressed when an anisotropic cloak $(2 \mu \mathrm{m} \times 2 \mu \mathrm{m})$ is placed on top of the bump. The grid lines correspond to the coordinate map of the transfinite transformation used to derive the EM parameters of the cloak. (C) The bump is cloaked for SPPs by means of an isotropic cloak. The lines show the quasiconformal coordinate grid, that determine the refractive index profile used in the cloak and depicted in (D). (E) Cloaking a 3D bump $1.25 \mu \mathrm{m} \times 1.25 \mu \mathrm{m} \times 200 \mathrm{~nm}$ for a SPP at $\lambda=600 \mathrm{~nm}$. The white lines correspond to the power flow streamlines. In (A), (B), (C) and (E) the color map shows the SPP magnetic field profile, $H_{y}$.

where $\Delta=\frac{\partial z^{\prime}}{\partial z}, f_{x z}=\frac{\partial z^{\prime}}{\partial x}$ and $f_{z z}=f_{x z}^{2}+\Delta^{2}$. When a medium with the prescribed anisotropic $\varepsilon^{\prime}$ and $\hat{\mu}^{\prime}$ is placed on top of the bump, a SPP smoothly travels through it without any scattering. This is confirmed by the 2D simulation illustrated in Figure 6 (B), where the magnetic field, $H_{y}$, of a SPP at $\lambda=700 \mathrm{~nm}$ going through the cloak is shown.

On the other hand, the same functionality can be achieved by means of an isotropic cloak. In order to design the isotropic ground-plane cloak, the transfinite transformation has to be replaced by a quasiconformal one. This kind of transformation is an approximation to a conformal mapping and it minimizes anisotropy too. It is generated by numerically solving Laplace's equation with sliding boundary conditions in the region defined by the cloak, which in this case is sized $6 \mu \mathrm{m} \times 2.5 \mu \mathrm{m}$. In contrast to the previous transformation, and as can be seen in panel (C), the new coordinate grid (gray lines) preserves the right-angles when the TO procedure is followed. This fact results in an isotropic refractive index $n(x, y)$ for the cloak illustrated in panel (D). The index profile is smooth, ranging from 0.82 to 1.38 , and it recovers the background value $n=1$ at the outer boundaries of the cloak. The wave fronts of SPPs traveling through the cloak follow the transformed grid while it smoothly goes through the bump without scattering, as illustrated for $\lambda=700 \mathrm{~nm}$ in panel (C).

Using the same tools, a ground-plane cloak can also be devised for hiding a 3D bump on a metal surface. Let us consider the bump shown in Figure 6 (E), which is described by $z^{\prime}=z+h_{0}\left(1-\frac{z}{h}\right) \cos ^{2}\left(\frac{2 \pi}{l} x\right) \cos ^{2}\left(\frac{2 \pi}{l} y\right)$. As a proof of principle we present an anisotropic 3D cloak, whose design involves a transfinite transformation analogous to the 2D case. According to the TO rules, the prescribed material parameters are the following:
$\hat{\varepsilon}^{\prime}=\hat{\mu}^{\prime}=\frac{1}{\Delta}\left(\begin{array}{ccc}1 & 0 & f_{x z} \\ 0 & 1 & f_{y z} \\ f_{x z} & f_{y z} & f_{z z}\end{array}\right)$

where $\Delta=\frac{\partial z^{\prime}}{\partial z}, f_{x z}=\frac{\partial z^{\prime}}{\partial x}, f_{y z}=\frac{\partial z^{\prime}}{\partial y}$ and $f_{z z}=f_{x z}{ }^{2}+f_{y z}{ }^{2}+\Delta^{2}$. The performance of a cloak with $l=1.25 \mu \mathrm{m}$ and $h_{0}=200 \mathrm{~nm}$ operating at $\lambda=600 \mathrm{~nm}$ can be seen in panel (E). The SPP magnetic field (color map) and power flow lines (in white) confirm the effectiveness of this plasmonic device. As in the 2D geometry, the ground-plane cloak completely suppresses the scattering from the bump.

\subsection{Plasmonic Luneburg lens}

Finally, we present as a last example two designs for a plasmonic Luneburg lens for SPPs propagating on a metal surface. A planar Luneburg lens is a circle of radius $R$ characterized by the following refractive index:

$n(r)=\sqrt{2-(r / R)^{2}}$

where $r$ is the distance to the center of the lens. This index profile focuses an incident SPP to a point in the perimeter of the lens on the opposite side of the circle. In a first design of the plasmonic Luneburg lens, let us assume a metamaterial that reproduces the refractive index $n(r)$. As sketched in Figure 7 (A), the metamaterial is a cylinder of radius $R$ placed on top of a gold surface with a height that needs to be larger than the SPP vacuum decay length, similar to the previous examples. Panel (B) demonstrates a plasmonic Luneburg lens of radius $R=2.5 \mu \mathrm{m}$ operating at $\lambda=800 \mathrm{~nm}$ when the spatial variation of the index $n(r)$ [shown in (C)] is implemented in the metamaterial region. The SPP electric field and power 
A

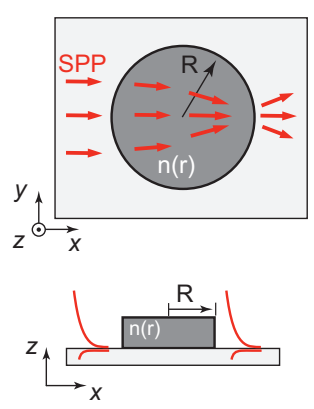

C

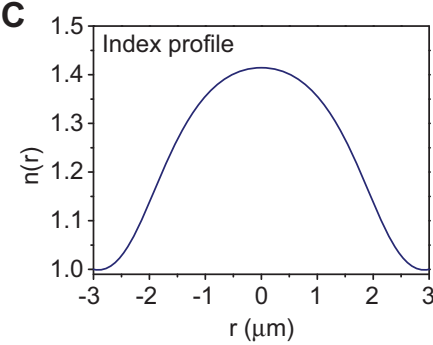

E
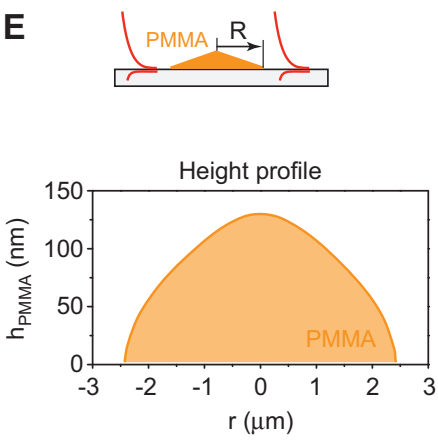

B

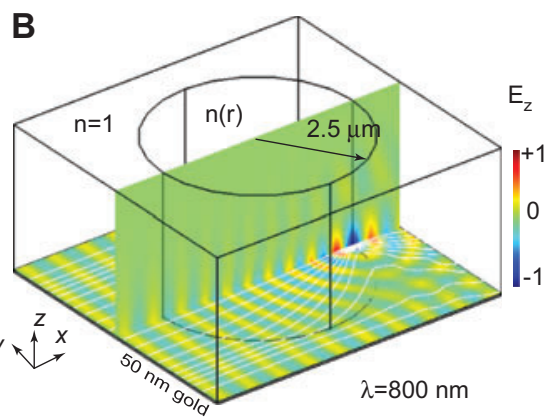

D

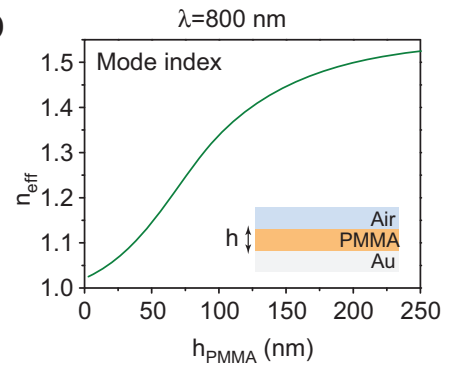

$\mathbf{F}$

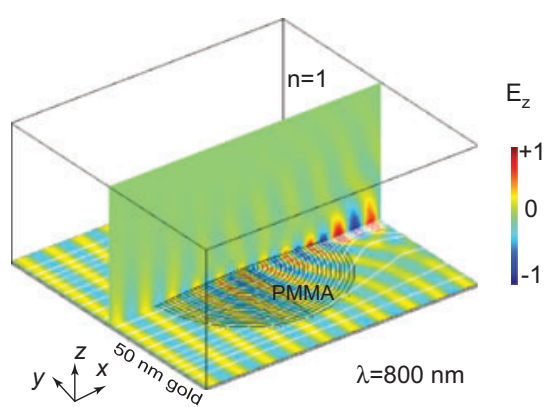

Figure 7 A plasmonic Luneburg lens is used to focus an incident SPP. (A) Schematic of a lens of radius $R$ in top and side views. The transformation medium is a cylinder of radius $R$ with height larger than the SPP decay length in the dielectric side of the interface. (B) Full-wave simulation of a lens for a SPP at $\lambda=800 \mathrm{~nm}$. The radius of the lens is $R=2.5 \mu \mathrm{m}$ and its height is $3 \mu \mathrm{m}$. (C) Refractive index as a function of the distance to the center of the lens, $r$, for $R=2.5 \mu \mathrm{m}$. (D) Relation between the SPP mode index at $\lambda=800 \mathrm{~nm}$ and the height of a PMMA layer placed on a gold surface. (E) Sketch of a lens that consists of a PMMA disc on the gold surface and height of the layer as a function of the distance to the center of the lens. (F) 3D simulation of a PMMA layer shaped according to panel (E) acting as a Luneburg lens for SPPs at $\lambda=800 \mathrm{~nm}$. Both (B) and (F) show the SPP electric field, $E_{z}$.

flow lines obtained from 3D simulations show the focusing of SPPs traveling along an air-gold interface at the perimeter of the lens.

A different strategy for designing this plasmonic element that involves the effective mode index of SPPs has been used to experimentally demonstrate the Luneburg lens [48]. Instead of spatially varying the optical properties of the dielectric, the height of a thin dielectric layer on top of the metal is tapered. The index profile given by Eq. 10 is reproduced by the effective mode index of the SPP. The mode index for an air/PMMA/gold structure for SPPs at $\lambda=800 \mathrm{~nm}$ is plotted in Figure 7 (D) as a function of the PMMA height, $h$. In a dielectric/dielectric/metal structure with electric permittivities $\varepsilon_{1}$, $\varepsilon_{2}$ and $\varepsilon_{3}$, respectively, SPPs at frequency $\omega$ propagate with an effective mode index $n_{\text {eff }}=k / k_{0}$ determined by the parallel wave-vector $k$. Here, $k_{0}=\omega^{2} / c^{2}$ and $c$ stands for light speed. The SPP dispersion relation is given by: $\tanh \left(k_{2} \varepsilon_{2} h\right)=\frac{k_{1} k_{2}+k_{2} k_{3}}{k_{2}^{2}+k_{1} k_{3}}$

where

$k_{i}=\frac{\sqrt{k^{2}-\varepsilon_{i} k_{0}^{2}}}{\varepsilon_{i}},(i=1,2,3)$

In our situation, $\varepsilon_{1}=1, \varepsilon_{2}=2.19$ and $\varepsilon_{3}=\varepsilon_{A u}$. For PMMA thicknesses up to $250 \mathrm{~nm}$, we see in panel (B) that the index varies between 1 and 1.5 , a range that is enough to reproduce the index required by the Luneburg lens. The plasmonic device, sketched in panel (E), is designed by calculating the PMMA's height at which the SPP effective mode index satisfies Eq. 10. This results in a variation of the PMMA height between 0 at the perimeter of the lens and $130 \mathrm{~nm}$ at the center. A 
numerical simulation of this lens can be seen in Figure 7 (F). A PMMA disk of $2.5 \mu \mathrm{m}$ radius with a height modulated according to panel (E) was placed on top of a gold surface. The SPP field is smoothly focused at the perimeter of the lens in a subwavelength spot. This design of the plasmonic lens potentially allows for a broad band performance, as it only involves dielectric materials with a very low dispersion.

These different examples, illustrate the power of transformation plasmonics to fully control SPP and design complex functionalities that would not be achievable otherwise. However, the experimental implementation of such control is conditioned by the possibility to match the required structural parameters. In the next section, we discuss recent experimental advances towards the realization of complex SPP functionalities giving special emphasis on the different fabrication strategies.

\section{Transformation plasmonics for real: experimental implementation}

\subsection{Fabrication and optical characterization methods}

The experimental implementation of Transformation Plasmonics at optical frequencies requires engineering on the subwavelength scale the effective mode index $n_{\text {eff }}$ of the propagating SPPs. In practice, this can be achieved in two different ways:

The first approach is based on a discrete patterning of a dielectric material lying on top of the flat metal film. This leads to an artificial- or meta-medium having a mean refractive index that affects $n_{\text {eff }}$ of the SPPs as shown by the blue curve in Figure 8. The elementary unit can either be an air hole in a continuous dielectric film as originally proposed in [35] for optical waveguides or a dielectric protrusion in air [85]. In this case, the local refractive index is spatially adjusted by controlling the units size and/or density [see Figure 9 (C)

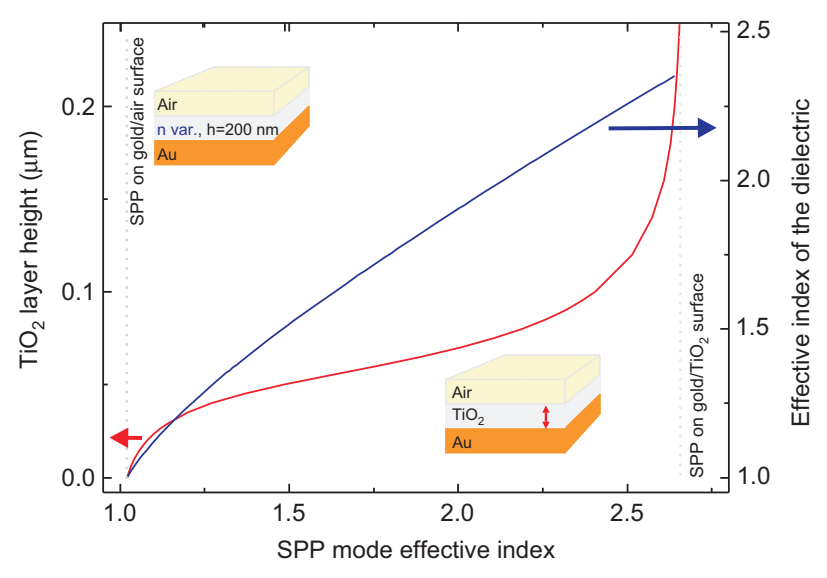

Figure 8 The SPP mode effective index can be controlled either by adjusting the thickness of the dielectric layer or the effective refractive index of an artificial medium, for instance formed by dielectric protrusions. The refractive index of the surrounding and the one of the dielectric load limits the accessible range of $n_{\text {eff }}$ and (D)]. In practice, this is achieved using standard fabrication tools such as electron-beam lithography and Focused Ion Beam (FIB). The minimum size of the elementary unit as well as the minimum distance between adjacent units determine the smoothness of the actual $n_{\text {eff }}$ profile. Maximizing the smoothness is crucial as it has direct impact on the back reflection and scattering of the incident SPP. Such parasitic reflection and scattering would for instance directly compromise the effciency of an invisibility cloak or carpet.

Alternatively, the desired $n_{\text {eff }}$ landscape can be obtained by gradually varying the thickness of a continuous dielectric load [13] as shown by the red curve in Figure 8. In their recent work, Zentgraf et al. [48] used 3D electron beam lithography to gradually shape a PMMA load on gold into a patch that fulfills the requirements of a Eaton and Luneburg plasmonic lens [Figure 9 (A-B)].

In both approaches, the accessible range for the effective mode index $n_{\text {eff }}$ is determined by the dielectric properties of the surrounding media and the dielectric being used. While the use of high refractive index materials a priori gives more flexibility, the propagation length of SPPs $\Lambda_{S P P}=1 /\left(2 \operatorname{Im}\left[n_{\text {eff }} k_{0}\right]\right)$ decreases for increasing values of $n_{\text {eff }}$, thereby adding further constrains in the design.

Because SPPs are surface waves, bound to the metal surface, the optical characterization of plasmonic functionalities requires suitable methods capable to monitor, either directly or indirectly, the interaction of the incident SPP with the metasurface. The simplest approach consists in measuring input and output signals without directly monitoring the interaction region $[35,102]$. Such a scheme can for instance be implemented by including input and output grating couplers at strategic locations of the sample. However, it is blind to what actually occurs in the interaction region and does not apply to any functionality. Further insight into the metasurface properties can be gained by employing scanning near-field optical microscopy (SNOM) [103] which can directly map with a sub-wavelength spatial resolution the optical near-field intensity of plasmon fields at any point of the metasurface. Special attention is though required to prevent significant influence of the scanning probe on the intrinsic properties of the structure under study [103-105]. Alternatively to SNOM, Leakage Radiation Microscopy offers a simpler and faster characterization as long as $n_{\text {eff }}$ is smaller than the refractive index of the substrate. Under this condition, SPPs are subjected to leakage radiation into the substrate. Although diffraction limited, collecting this leakage radiation offers a wide field, probe-less and quantitative method [106-108] to map the SPP propagation at the metasurface.

\subsection{Experimental implementations}

In the following we briefly discuss recent experiments that illustrate the power of transformation plasmonics to design complex plasmonic functionalities.

4.2.1. Broadband plasmonic invisibility carpet at visible frequencies The first example tackles the concept of invisibility carpet and demonstrates experimentally that 
A
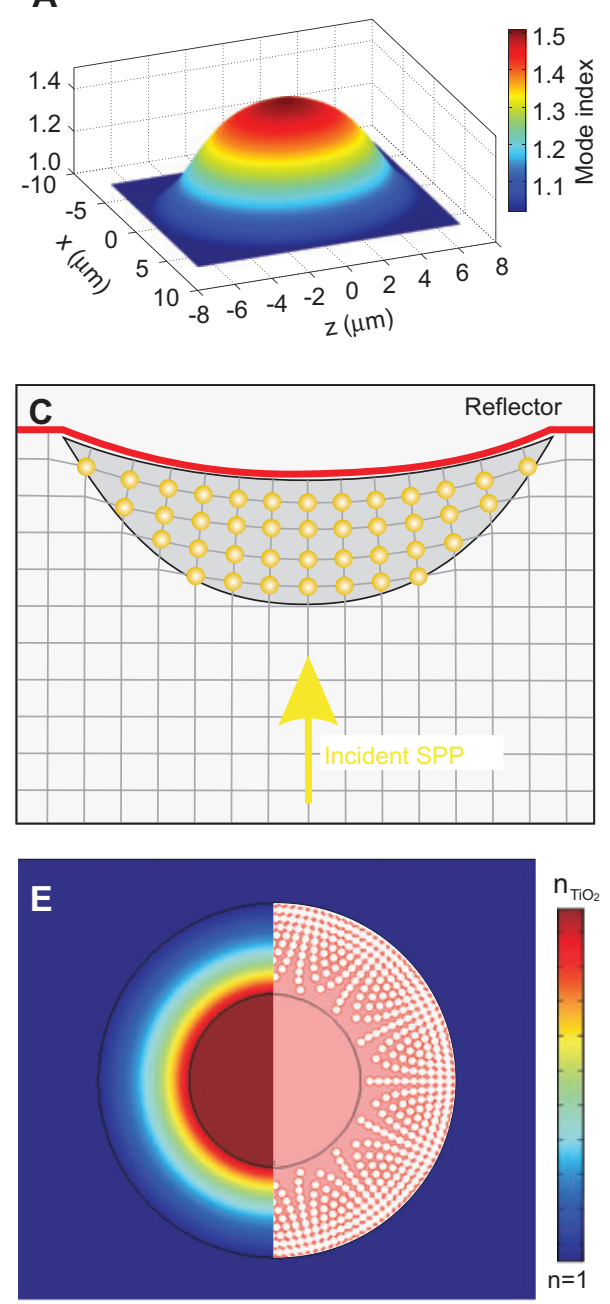
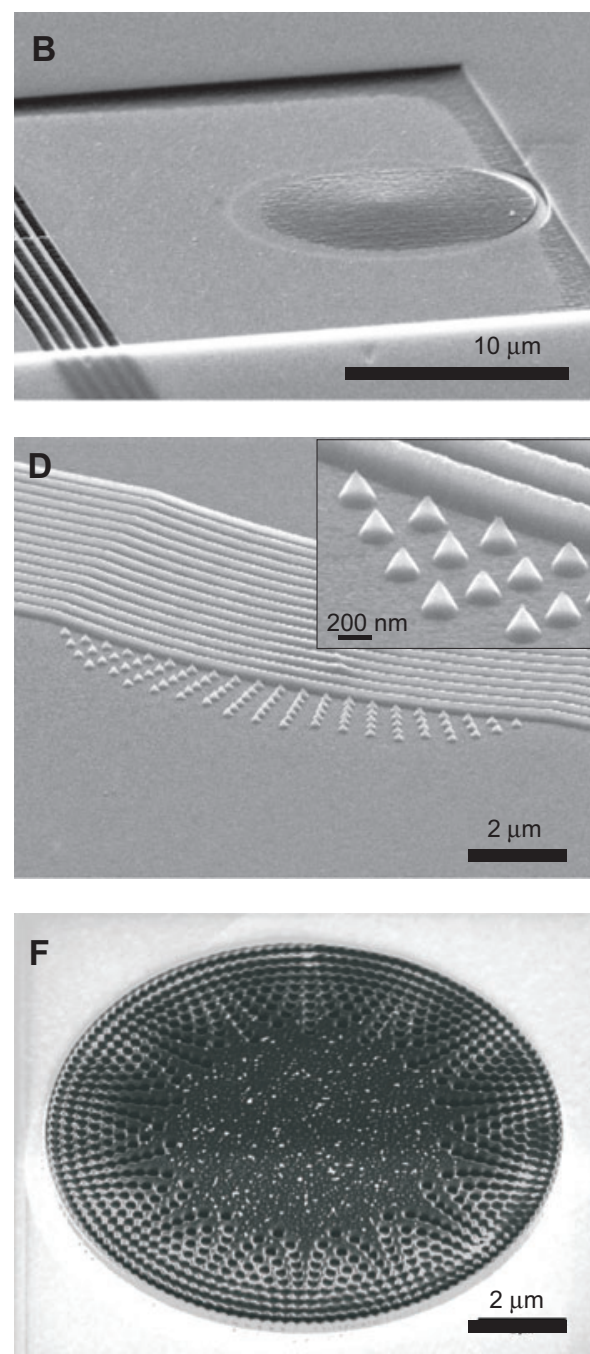

Figure 9 The mode effective index for SPPs can be controlled via the height (A,B) of the dielectric layer or the density and size of particles $(\mathrm{C}, \mathrm{D})$ or holes $(\mathrm{E}, \mathrm{F})$. The calculated index profile for a Luneburg lens (A) has been realized by 3D lithography of the PMMA on top of gold layer. (A,B) reprinted by courtesy of X. Zhang from Ref. [48]. (D) Small cones made of $\mathrm{TiO}_{2}$ have been placed at the nodes of the carpet cloak (C). The high density of holes at the outer rim gradually changes towards the inner region of the SPP concentrator shown in (F) corresponding to index profile shown (E).

an object can be concealed from a SPP propagating along a gold/air interface at visible frequencies, as we numerically demonstrated in Figure 3.

In order to meet experimentally the parameters of the simulations, a flat gold surface was structured with $\mathrm{TiO}_{2}$ protrusions. The $\mathrm{TiO}_{2}$ pillars forming the crescentmoon-like carpet were first fabricated on top of a 60-nm thin Au film by combining electron-beam lithography and reactive-ion etching. In a second lithography step, a curved Bragg-type reflector [formed by 15 gold lines (section=150 $\mathrm{nm} \times 150 \mathrm{~nm}$ ) periodically separated by half of the SPP wavelength] was added, acting as the object to be hidden behind the carpet Figure 9 (C) and (D). Each of the small cones of $\mathrm{TiO}_{2}$ are placed at the nodes of a quasi-conformal grid, what allows to minimize the required anisotropy of the metric tensor associated with the carpet shown in Figure 3. This simple trick greatly simplifies the experimental design.
In the optical experiment, the SPPs were launched by focusing the incident beam from a CWTi:Sapph laser on a defect line placed $44 \mu \mathrm{m}$ away from the reflector. Taking advantage of the asymmetry of the dielectric environment on both side of the gold film, the leakage radiation from the SPPs at the gold/air interface was collected using a high-numerical aperture objective to map the SPP fields. Additionally for the sake of clarity, spatial filtering in the conjugated Fourier-plane was performed to suppress the direct transmitted light from the excitation spot and scattered light in order to isolate the carpet properties.

Leakage radiation microscopy (LRM) was first used to map the distribution of $\lambda=800 \mathrm{~nm}$ SPPs propagating at the gold/air interface and interacting with the different structures fabricated at the gold surface. In the case of a bare curved Bragg-reflector, the reflected SPPs propagate into different directions depending on their relative angle to the normal to the mirror lines [see Figure 10 (B)], thus leading to a curved 

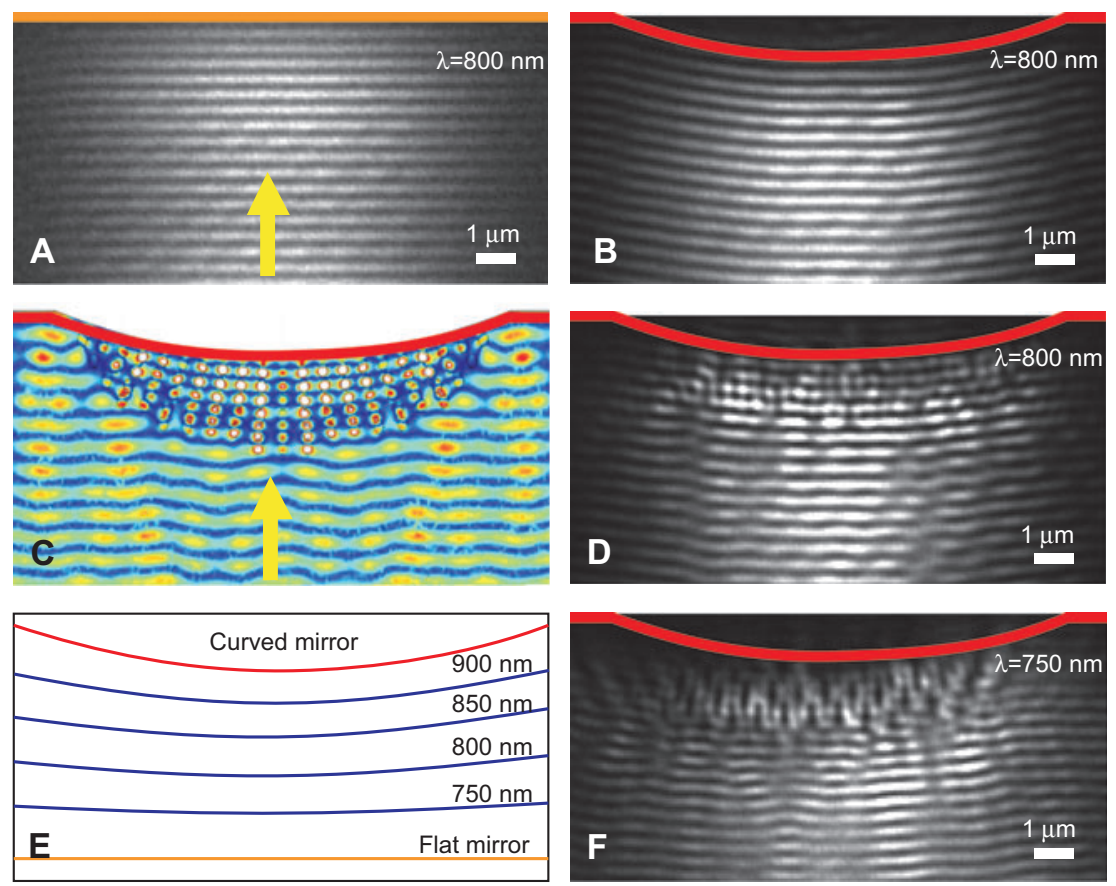

Figure 10 Broadband plasmonic invisibility carpet at optical frequencies: (A,B) Leakage radiation microscopy data showing the reflection of visible SPPs at $\lambda=800 \mathrm{~nm}$ on a flat (A) and bare curved Bragg mirror (B). (D,F) illustrate the effect of the invisibility carpet at two different wavelengths. (C) Numerical simulation associated with the experimental map (D). (E) displays the evolution of the experimental re-flected wavefront as a function of the incident wavelength, demonstrating the broadband operation over the 750-900 $\mathrm{nm}$ range.

wave front. Conversely, adding the crescent-moon-like $\mathrm{TiO}_{2}$ carpet re-establishes a fringe pattern with a nearly straight wave front [see Figure 10 (D)] similar to the case of a flat Bragg-mirror (A). The remaining small lateral modulations were attributed to imperfections in the manufacturing. Quantifying the modification in the wave front curvature induced by the presence of the crescent-moon-like $\mathrm{TiO}_{2}$ carpet and comparing the area under the numerically averaged curves with the curved obtained with the reference structure without carpet leads to a reduction by a factor 3.7 as shown in Figure 10 (E). Further, the experiment was repeated at different incident wavelengths across the range of the Ti:Sapph laser in order to test the broadband operation of the carpet. The data summarized in Figure 10 (E) show that the structure remains effcient between $750 \mathrm{~nm}$ and $850 \mathrm{~nm}$, demonstrating for the first time broadband invisibility in the true visible range.

4.2.2. Luneburg and Eaton plasmonic Lens Prior experimental studies demonstrated that the change in the effective refractive index $n_{\text {eff }}$ induced by patterning a metal surface with a dielectric load of constant height can be used to control the propagation of SPPs by exploiting the fact that the SPP propagation at the load boundaries fulfills the 2D equivalent of the Snell-Descartes laws. Through a suitable design of the load geometry, SPPs can thus be guided [12, 13, 15], reflected [109, 110] and focused [11]. Recent experiments by the Zhang's group [48] further pushed the analogy between 3D optical and plasmonic elements by using transformation plasmonics. By gradually varying the

height of a PMMA disk lying on top of a thin gold film using 3D e-beam lithography (see Figure 9), the authors first built a Luneburg plasmonic lens whose focal point lies on the perimeter of the lens. The fabricated structure was optically tested by combining fluorescence imaging and LRM. Their data, summarized in Figure 11, show a good agreement with their numerical simulation (A), efficient focusing in the near-infrared region of spectrum, between $\lambda=770$ and $840 \mathrm{~nm}$ (B). To demonstrate the versatility of their approach, the authors also successfully demonstrated the concept of Eaton plasmonic lens that bends the SPP through $90^{\circ}$.

A

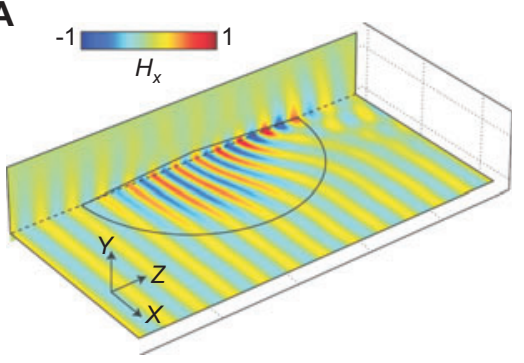

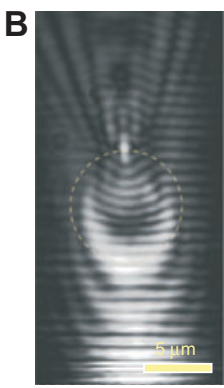

Figure 11 Plasmonic Luneburg Lens. (A) Simulation showing how incident SPPs are focused at a point at the outer perimeter of the Luneburg lens. (B) Leakage Radiation microscopy reveals the SPP focus at $\lambda=800 \mathrm{~nm}$. (A,B) reprinted by courtesy of $\mathrm{X}$. Zhang from Ref. [48]. 


\section{Conclusion and prospects}

Transformation plasmonics complements the existing tool box available to researchers in the field of plasmonics by providing further control over surface plasmons. Its full determinism potentially enables to tame plasmonic fields at will and this way design functionalities that were not reachable so far. First experimental realizations successfully demonstrated the practical feasibility of this new approach to control the propagation of SPPs at a patterned metal surface. Similarly to what is currently experienced in the field of metamaterials, a full exploitation of transformation plasmonics at optical frequencies remains though hindered by current limitations in nanofabrication. Beyond extending the range of plasmonic functionalities based on SPP and optimizing their effciency, a higher fabrication accuracy should enable to extend TO to the nanoscale light control based on Localized Surface Plasmons supported by 3D metallic nanostructures [93, 94, $111,112]$. Another important future development would consist in combining transformation plasmonics with recent advances in active plasmonics [113-115] by using active materials instead of passive dielectrics in order to introduce a dynamic control through an external electrical or optical signal.

\section{Acknowledgments}

This work was sponsored by the Spanish Ministry of Science under projects CSD2007-046-NanoLight.es and MAT2011-28581-C02-01. P.A.H. acknowledges financial support from the Spanish Ministry of Education through grant no. AP2008-00021. J.R. and R.Q. acknowledge financial support by the european commission through grant ERC-St Plasmolight (259196) and the Fundació Privada CELLEX.

\section{References}

[1] Abelès F, Lopez-Rios T. Surface polaritons at metal surfaces and interfaces. In: Agranovich VM and Mills DL, editors. Surface ploaritions. Amsterdam: North-Holland Publishing Company; 1982, pp. 239-74.

[2] Raether H. Surface plasmons. Berlin: Springer-Verlag; 1988.

[3] Zayats AV, Smolyaninov II, Maradudin AA. Nano-optics of surface plasmon polaritons. Phys Reports 2005;408:131-314.

[4] Weeber J-C, Krenn JR, Dereux A, Lamprecht B, Lacroute Y, Goudonnet JP. Near-field observation of surface plasmon polariton propagation on thin metal stripes. Phys Rev B 2001;64:045411-1-9.

[5] Barnes WL, Dereux A, Ebbesen TW. Surface plasmon subwavelength optics. Nature 2003;424:824-30.

[6] Bozhevolnyi SI, Volkov VS, Devaux E, Laluet J-Y, Ebbesen TW. Channel plasmon subwavelength waveguide components including interferometers and ring resonators. Nature 2006;440:508-11.

[7] Moreno E, Rodrigo SG, Bozhevolnyi SI, Martín-Moreno L, García-Vidal FJ. Guiding and focusing of electromagnetic fields with wedge plasmon polaritons. Phys Rev Lett 2008;100:023901.

[8] Gramotnev DK, Bozhevolnyi SI. Plasmonics beyond the diffraction limit. Nature Photon 2010;4:83-91.
[9] Stockman MI. Nanofocusing of optical energy in tapered plasmonic waveguides. Phys Rev Lett 2004;93:137404.

[10] Verhagen E, Polman A, Kuipers L. Nanofocusing in laterally tapered plasmonic waveguides. Opt Express 2008;16:45-57.

[11] Hohenau A, Krenn JR, Stepanov AL, Drezet A, Ditlbacher H, Steinberger B, Leitner A, Aussenegg FR. Dielectric optical elements for surface plasmons. Opt Lett 2005;30:893-5.

[12] Steinberger B, Hohenau A, Ditlbacher H, Aussenegg FR, Leitner A, Krenn JR. Dielectric stripes on gold as surface plasmon waveguides: bends and directional couplers. Appl Phys Lett 2007;91:081111.

[13] Holmgaard T, Bozhevolnyi SI. Theoretical analysis of dielectric-loaded surface plasmon-polariton waveguides. Phys Rev B 2007;75:245405.

[14] Krasavin AV, Zayats AV. Passive photonic elements based on dielectric-loaded surface plasmon polariton waveguides. Appl Phys Lett 2007;90:211101.

[15] Grandidier J, Massenot S, Colas des Francs G, Bouhelier A, Weeber J-C, Markey L, Dereux A, Renger J, González MU, Quidant R. Dielectricloaded surface plasmon polariton waveguides: figures of merit and mode characterization by image and Fourier plane leakage microscopy. Phys Rev B 2008;78:245419.

[16] Pendry JB, Martín-Moreno L, García-Vidal FJ. Mimicking surface plasmons with structured surfaces. Science 2004;305:847-8.

[17] Ramakrishna SA, Mandal P, Jeyadheepan K, Shukla N, Chakrabarti S, Kadic M, Enoch S, Guenneau S. Plasmonic interaction of visible light with gold nanoscale checkerboards. Phys Rev B 2011;84:245424.

[18] Wood JJ, Tomlinson LA, Hess O, Maier SA, FernándezDomínguez AI. Spoof plasmon polaritons in slanted geometries. Phys Rev B 2012;85:075441.

[19] Smolyaninov II, Hung YJ, Davis CC. Twodimensional metamaterial structure exhibiting reduced visibility at $500 \mathrm{~nm}$. Opt Lett 2008;33:1342-4.

[20] Smolyaninov II. Two-dimensional metamaterial optics. Laser Phys Lett 2010;7:259-69.

[21] Baumeier B, Leskova TA, Maradudin AA. Cloaking from surface plasmon polaritons by a circular array of point scatterers. Phys Rev Lett 2009;103:246803.

[22] Pendry JB, Schurig D, Smith DR. Controlling electromagnetic fields. Science 2006;312:1780-2.

[23] Leonhardt Ulf. Optical conformal mapping. Science 2006;312:1777-80.

[24] Kildishev AV, Cai W, Chettiar UK, Shalaev VM. Transformation optics: approaching broadband electromagnetic cloaking. New J Phys 2008;10:115029.

[25] Li J, Pendry JB. Hiding under the carpet: a new strategy for cloaking. Phys Rev Lett 2008;101:203901.

[26] Crudo RA, O’Brien JG. Metric approach to transformation optics. Phys Rev A 2009;80:033824.

[27] Kwon D-H, Werner DH. Transformation electromagnetics: an overview of the theory and applications. Antennas and Propagation Magazine, IEEE 2010;52:24-46.

[28] Gallina I, Castaldi G, Galdi V, Alu A, Engheta N. General class of metamaterial transformation slabs. Phys Rev B 2010;81:125124.

[29] Popa B-I, Cummer SA. Design of layered transformation-optics devices of arbitrary shape. Phys Rev A 2010;82:033837.

[30] Guenneau S, McPhedran RC, Enoch S, Movchan AB, Farhat M, Nicorovici N-AP. The colours of cloaks. J Opt 2011;13:024014. 
[31] Schurig D, Mock JJ, Justice BJ, Cummer SA, Pendry JB, Starr, AF, Smith DR. Metamaterial electromagnetic cloak at microwave frequencies. Science 2006;314:977-80.

[32] Cai W, Chettiar UK, Kildishev AV, Shalaev VM. Optical cloaking with metamaterials. Nature Photon 2007;1:224-7.

[33] Jiang WX, Chin JY, Cui TJ. Anisotropic metamaterial devices. Materials Today 2009;12:26-33.

[34] Gabrielli LH, Cardenas J, Poitras CB, Lipson M. Silicon nanostructure cloak operating at optical frequencies. Nature Photon 2009;3:461-3.

[35] Valentine J, Li J, Zentgraf T, Bartal G, Zhang X. An optical cloak made of dielectrics. Nature Mater 2009;8:568-71.

[36] Kanté B, Germain D, de Lustrac A. Experimental demonstration of a nonmagnetic metamaterial cloak at microwave frequencies. Phys Rev B 2009;80:201104.

[37] Semouchkina E, Werner DH, Semouchkin GB, Pantano C. An infrared invisibility cloak composed of glass. Appl Phys Lett 2010;96:233503.

[38] Zhai YB, Ping XW, Jiang WX, Cui TJ. Finite-element analysis of three-dimensional axisymmetrical invisibility cloaks and other metamaterial devices. Commun Comput Phys 2010;8:823-34.

[39] Ergin T, Stenger N, Brenner P, Pendry JB, Wegener M. Threedimensional invisibility cloak at optical wavelengths. Science 2010;328:337-9.

[40] Wu K, Cheng Q, Wang GP. Fourier optics theory for invisibility cloaks. J Opt Soc Am B 2011;28:1467-74.

[41] Ergin T, Fischer J, Wegener M. Optical phase cloaking of 700 $\mathrm{nm}$ light waves in the far field by a threedimensional carpet cloak. Phys Rev Lett 2011;107:173901.

[42] Perczel J, Tyc T, Leonhardt U. Invisibility cloaking without superluminal propagation. New J Phys 2011;13:083007.

[43] Gharghi M, Gladden C, Zentgraf T, Liu Y, Yin, X, Valentine J, Zhang X. A carpet cloak for visible light. Nano Lett 2011;11:2825-8.

[44] Zhang J, Liu L, Luo Y, Zhang S, Mortensen NA. Homogeneous optical cloak constructed with uniform layered structures. Opt Express 2011;19:8625-31.

[45] Ma YG, Ong CK, Tyc T, Leonhardt U. An omnidirectional retroreflector based on the transmutation of dielectric singularities. Nature Materials 2009;8:639-42.

[46] Kundtz N, Smith DR. Extreme-angle broadband metamaterial lens. Nature Materials 2010;9:129-32.

[47] Turpin JP, Massoud AT, Jiang ZH, Werner PL, Werner DH. Conformal mappings to achieve simple material parameters for transformation optics devices. Opt Express 2010;18:244-52.

[48] Zentgraf T, Liu Y, Mikkelsen MH, Valentine J, Zhang X. Plasmonic Luneburg and Eaton lenses. Nature Nanotech 2011;6:151-5.

[49] Rahm M, Cummer SA, Schurig D, Pendry JB, Smith DR. Optical design of reflectionless complex media by finite embedded coordinate transformations. Phys Rev Lett 2008;100:063903.

[50] Chen H, Chan CT. Electromagnetic wave manipulation by layered systems using the transformation media concept. Phys Rev B 2008;78:054204.

[51] Roberts DA, Rahm M, Pendry JB, Smith DR. Transformationoptical design of sharp waveguide bends and corners. Appl Phys Lett 2008;93:251111.

[52] Rahm M, Roberts DA, Pendry JB, Smith DR. Transformationoptical design of adaptive beam bends and beam expanders. Opt Express 2008;16:11555-67.

[53] Landy NI, Padilla WJ. Guiding light with conformal transformations. Opt Express 2009;17:14872-9.
[54] Vasic B, Gajic R, Isic G, Hingerl K. Confined metamaterial structures based on coordinate transformations. Acta Phys Polonica A 2009;116:96.

[55] Greenleaf A, Kurylev Y, Lassas M, Uhlmann G. Electromagnetic wormholes and virtual magnetic monopoles from metamaterials. Phys Rev Lett 2007;99:183901.

[56] Kadic M, Dupont G, Guenneau S, Enoch S. Plasmonic wormholes: defeating the early bird. arXiv:1102.2372v1, 2011.

[57] Chen H, Chan CT, Liu S, Lin Z. A simple route to a tunable electromagnetic gateway. New J Phys 2009;11:083012.

[58] Luo X, Yang T, Gu Y, Chen H, Ma H. Conceal an entrance by means of superscatterer. Appl Phys Lett 2009;94:223513.

[59] Leonhardt U, Piwnicki P. Optics of nonuniformly moving media. Phys Rev A 1999;60:4301-12.

[60] Rahm M, Schurig D, Roberts DA, Cummer SA, Smith DR, Pendry JB. Design of electromagnetic cloaks and concentrators using form-invariant coordinate transformations of maxwells equations. Photonics Nanostruct Fundam Appl 2008;6:87-95.

[61] Genov DA, Zhang S, Zhang X. Mimicking celestial mechanics in metamaterials. Nature Phys 2009;5:687-692.

[62] Narimanov EE, Kildishev AV. Optical black hole: broadband omnidirectional light absorber. Appl Phys Lett 2009;95:041106.

[63] Argyropoulos C, Kallos E, Hao Y. FDTD analysis of the optical black hole. J Opt Soc Am B 2010;27:2020-25.

[64] Chen H, Miao R-X, Li M. Transformation optics that mimics the system outside a Schwarzschild black hole. Opt Express 2010;18:15183-8.

[65] Cheng Q, Cui, TJ, Jiang WX, Cai BG. An omnidirectional electromagnetic absorber made of metamaterials. New J Phys 2010;12:063006.

[66] Kadic M, Dupont G, Chang T-M, Guenneau S, Enoch S. Curved trajectories on transformed metal surfaces: beam-splitter, invisibility carpet and black hole for surface plasmon polaritons. Photonics Nanostruct Fundam Appl 2011;9:302-7.

[67] Lai Y, Ng J, Chen HY, Han DZ, Xiao JJ, Zhang Z-Q, Chan CT. Illusion optics: the optical transformation of an object into another object. Phys Rev Lett 2009;102:253902.

[68] Zolla F, Guenneau S, Nicolet A, Pendry JB. Electromagnetic analysis of cylindrical invisibility cloaks and the mirage effect. Opt Lett 2007;32:1069-71.

[69] Diatta A, Guenneau S. Non singular cloaks allow mimesis. J Optic 2011;13:24012.

[70] Nicolet A, Zolla F, Geuzaine C. Generalised cloaking and optical polyjuice. arXiv:0909.0848v1, 2009.

[71] Salandrino A, Engheta N. Far-field subdiffraction optical microscopy using metamaterial crystals: theory and simulations. Phys Rev B 2006;74:075103.

[72] Liu Z, Lee H, Xiong Y, Sun C, Zhang X. Far-field optical hyperlens magnifying sub-diffraction-limited objects. Science 2007;315:1686.

[73] Smolyaninov II, Hung Y-J, Davis CC. Magnifying superlens in the visible frequency range. Science 2007;315:1699-701.

[74] Kildishev AV, Narimanov EE. Impedancematched hyperlens. Opt Lett 2007;32:3432-4.

[75] Kildishev AV, Shalaev VM. Engineering space for light via transformation optics. Opt Lett 2008;33:43-5.

[76] Engheta N, Ziolkowski RW. Metamaterials: physics and engineering explorations. Wiley-IEEE Press; 2006.

[77] Ramakrishna SA. Physics of negative refractive index materials. Rep Prog Phys 2005;68:449.

[78] Ramakrishna SA, Grzegorczyk TM. Physics and applications of negative refractive index materials. CRC Press; 2008. 
[79] Maier SA. Plasmonics: fundamentals and applications. Springer Verlag; 2007.

[80] Alu A, Engheta N. Achieving transparency with plasmonic and metamaterial coatings. Phys Rev E 2005;72:016623.

[81] Nicorovici NA, Milton GW, McPhedran RC, Botten LC. Quasistatic cloaking of two-dimensional polarizable discrete systems by anomalous resonance. Opt Express 2007;15:6314-23.

[82] Kadic M, Guenneau S, Enoch S. Transformational plasmonics: cloak, concentrator and rotator for SPPs. Opt Express 2010;18:12027-32.

[83] Huidobro PA, Nesterov ML, Martín-Moreno L, GarcíaVidal FJ. Transformation optics for plasmonics. Nano Lett 2010;10:1985-90.

[84] Liu Y, Zentgraf T, Bartal G, Zhang X. Transformational plasmon optics. Nano Lett 2010;10:1991-7.

[85] Renger J, Kadic M, Dupont G, A imovi SS, Guenneau S, Quidant R, Enoch S. Hidden progress: broadband plasmonic invisibility. Opt Express 2010;18:15757-68.

[86] Huidobro PA, Nesterov ML, Martín-Moreno L, García-Vidal FJ. Moulding the flow of surface plasmons using conformal and quasiconformal mappings. New J Phys 2011;13:033011.

[87] Kadic M, Dupont G, Guenneau S, Enoch S. Controlling surface plasmon polaritons in transformed coordinates. J Mod Opt 2011;58:994-1003.

[88] Kadic M, Guenneau S, Enoch S, Ramakrishna SA. Plasmonic space folding: focusing surface plasmons via negative refraction in complementary media. ACS Nano 2011;5:6819-25.

[89] Vakil A, Engheta N. Transformation optics using graphene. Science 2011;332:1291-4.

[90] Aubry A, Lei DY, Fernández-Domínguez AI, Sonnefraud Y, Maier SA, Pendry JB. Plasmonic light-harvesting devices over the whole visible spectrum. Nano Lett 2010;10:2574-9.

[91] Aubry A, Lei DY, Maier SA, Pendry JB. Conformal transformation applied to plasmonics beyond the quasistatic limit. Phys Rev B 2010;82:205109.

[92] Fernández-Domínguez AI, Maier SA, Pendry JB. Collection and concentration of light by touching spheres: a transformation optics approach. Phys Rev Lett 2010;105:266807.

[93] Aubry A, Lei DY, Maier SA, Pendry JB. Interaction between plasmonic nanoparticles revisited with transformation optics. Phys Rev Lett 2010;105:233901.

[94] Aubry A, Lei DY, Maier SA, Pendry JB. Plasmonic hybridization between nanowires and a metallic surface: a transformation optics approach. ACS Nano 2011;5:3293-308.

[95] Fernández-Domínguez AI, Wiener A, García- Vidal FJ, Maier SA, Pendry JB. Transformation-optics description of nonlocal effects in plasmonic nanostructures. Phys Rev Lett 2012;108:106802.

[96] Nicolet A, Remacle J-F, Meys B, Genon A, Legros W. Transformation methods in computational electromagnetism. J Appl Phys 1994;75:6036-8.

[97] Berenger JP. A perfectly matched layer for the absorption of electromagnetic waves. J Comput Phys 1994;114:185-200.
[98] Ward AJ, Pendry JB. Refraction and geometry in maxwell's equations. J Mod Opt 1996;43:773-93.

[99] Zolla F, Renversez G, Nicolet A, Kuhlmey B, Guenneau S, Felbacq D. Foundations of photonic crystal fibres. Imperial College Press; 2005.

[100] Greenleaf A, Lassas M, Uhlmann G. Anisotropic conductivities that cannot detected in EIT. Physiol Meas 2003;24:413-20.

[101] COMSOL. Multiphysics 3.5a. http://www.comsol.com.

[102] Lezec HJ, Dionne JA, Atwater HA. Negative refraction at visible frequencies. Science 2007;316:430-2.

[103] Novotny L, Hecht B. Principles of nano-optics. Cambridge University Press; 2006.

[104] Hecht B, Bielefeldt H, Inouye Y, Pohl DW, Novotny L. Facts and artifacts in near-field optical microscopy. J Appl Phys 1997;81:2492-8.

[105] Vogelgesang R, Dmitriev A. Real-space imaging of nanoplasmonic resonances. Analyst 2010;135:1175-81.

[106] Bouhelier A, Huser Th, Tamaru H, Güntherodt H-J, Pohl DW, Baida FI, Van Labeke D. Plasmon optics of structured silver films. Phys Rev B 2001;63:155404-1-9.

[107] Drezet A, Hohenau A, Koller D, Stepanov A, Ditlbacher H, Steinberger B, Aussenegg FR, Leitner A, Krenn JR. Leakage radiation microscopy of surface plasmon polaritons. Mater Sci Eng B 2008;149:220.

[108] Hohenau A, Krenn JR, Drezet A, Mollet O, Huant S, Genet C, Stein B, Ebbesen TW. Surface plasmon leakage radiation microscopy at the diffraction limit. Opt Express 2011;19:25749-62.

[109] Ditlbacher H, Krenn JR, Schider G, Leitner A, Aussenegg FR. Two-dimensional optics with surface plasmon polaritons. Appl Phys Lett 2002;81:1762-4.

[110] Randhawa S, González MU, Renger J, Enoch S, Quidant R. Design and properties of dielectric surface plasmon bragg mirrors. Opt Express 2010;18:14496-510.

[111] Pacifici D, Lezec HJ, Atwater HA. All-optical modulation by plasmonic excitation of CdSe quantum dots. Nature Photon 2007;1:402-6.

[112] Krasavin AV, Randhawa S, Bouillard J-S, Renger J, Quidant R, Zayats AV. Optically-programmable nonlinear photonic component for dielectric-loaded plasmonic circuitry. Opt Express 2011;19:25222-9.

[113] Berini P, De Leon I. Surface plasmonpolariton amplifiers and lasers. Nature Photon 2012;6:16-24.

[114] Seidel J, Grafström S, Eng L. Stimulated emission of surface plasmons at the interface between a silver film and an optically pumped dye solution. Phys Rev Lett 2005;94:177401-1-4.

[115] Krasavin AV, Vo TP, Dickson W, Bolger PM, Zayats AV. Allplasmonic modulation via stimulated emission of copropagating surface plasmon polaritons on a substrate with gain. Nano Lett 2011;11:2231-5.

Received February 29, 2012; accepted May 4, 2012 